\title{
Unifying cognitive aging: From neuromodulation to representation to cognition
}

\author{
Shu-Chen $\mathrm{Li}^{\mathrm{a}, *}$, Ulman Lindenberger ${ }^{\mathrm{a}}$, Peter A. Frensch ${ }^{\mathrm{b}}$ \\ ${ }^{\mathrm{a}}$ Max Planck Institute for Human Development, Center of Lifespan Psychology Lentzeallee 94, \\ D-14195 Berlin, Germany \\ ${ }^{\mathrm{b}}$ Humboldt-University, Psychology Department, Hausvogteiplatz 5-7 D-10117 Berlin, Germany
}

Accepted 13 January 2000

\begin{abstract}
An integrative theory relating cognitive aging deficits observed at the behavioral level with age-related deficiency in neuromodulation causing less distinctive cortical representations is tested in a series of neural network simulations. Age-related attenuation of catecholaminergic function is simulated by lowering the mean gain of the processing unit, which subsequently reduces responsivity and raises intra-network activation variability. Age differences in learning rate, asymptotic performance, interference susceptibility, complexity cost, intra- and interindividual variability, and ability dedifferentiation can all be modeled. Together, the simulations illustrate catecholamine's role in regulating the fidelity of neural information processing and subsequent effects leading to cognitive aging deficits. (C) 2000 Elsevier Science B.V. All rights reserved.
\end{abstract}

Keywords: Cognitive aging; Neuromodulation; Catecholamine; Neural networks; Gain parameter

\section{Introduction}

Declines in basic mechanisms of cognition pervade the aging process. Behaviorally, age differences in processing speed [26], learning rate [16], asymptotic performance $[4,27]$, interference susceptibility [16,18], complexity cost [17] and intra-individual [11] as well as inter-individual variability [23] are robust effects. Biologically, brain

\footnotetext{
*Corresponding author. Tel.: + 49-30-82406305; fax: + 49-30-8249939.

E-mail address: shuchen@mpib-berlin.mpg.de (S.-C. Li).
} 
aging involves neuroanatomical and neurochemical changes [29]. Accounts postulated at either the information-processing level assuming age-related reduction in general processing resources [25] or at the biological level hypothesizing age-related increase in neural noise [34] were proposed as mediating factors for cognitive deficits observed behaviorally. However, thus far data and explanations of cognitive aging deficits have been mostly confined within one (or two) of these levels.

As an attempt to facilitate integration, we offer a cross-level theoretical conjecture aiming at integrating findings of age-related decrements in catecholaminergic function, the functional properties of catecholaminergic modulation, catecholaminergic effects on neural information processing, and various benchmark behavioral manifestations of cognitive aging. Empirical evidences from different levels are reviewed as we unfold and evaluate our conjecture.

\section{Aging, neuromodulation and information processing}

The relationship between cognitive aging and age-related deficiency in neuromodulation has recently become an important topic in aging research. Given their roles in modulating prefrontal cortical (PFC) cognitive functions [2], age-related depletion of catecholamine, consisting of dopamine (DA) and its metabolic products, norepinephrine (NE) and epinephrine, is of specific interest. There is consensus of age-related decline in dopaminergic function in the striatum, basal ganglia, and prefrontal cortex (PFC) both in terms of transmitter contents and binding mechanisms $[8,12,13,29]$. There is also evidence for functional relationships between agerelated deficits in the catecholaminergic system and age-related decrements in different aspects of cognitive functioning, such as processing speed [21] and working memory [28]. In the light of these findings, some researchers have suggested that catecholamine mechanisms play important roles in cognitive aging $[1,12,33]$.

Fig. 1. (A) The logistic activation function at different values of $G$. The logistic function is strictly for all value of $G>0$ and all values of the bias. Physiological evidence suggesting that the function relating the strength of an input signal to a neuron's firing rate is sigmoid, with its steepest slope around the baseline firing rate. In addition, small increments in excitatory signals produce greater changes in firing frequency than those produced by the same amount of increment in inhibitory signals. These properties can be jointly captured by the logistic function with a constant negative bias $[5,10]$. The bias of the logistic function is set to -1.0 . Reducing $G$ flattens the activation function such that a unit becomes less responsive: with a decrease in $G$, the effect of the net input is decreased both for excitatory and inhibitory signals. Subsequently, a $G$-reduced unit is also less responsive to the difference between signals. For instance, two different signals, +1 and -1 , produce a much greater difference in output activation when $G=1.0(0.5 \mathrm{vs}$. 0.12 ) than when $G=0.1$ ( 0.29 vs. 0.25 ); and when $G=0$ (only as an example of the limiting case) the unit's response remains always at its baseline activation. Given a same range of variability in $G$ s, reducing mean $G$ also increases the variability in output activation. (B) Reducing $G$ and the temporal variability of activation. Reducing mean $G$ ( 0.8 and 0.3 for the "young" and "old" networks, respectively) increases the temporal variability of a unit's response to an identical signal (was set to 4.0) across 1000 trials. (C) Internal activation patterns across five intermediate units of one young (mean $G=0.8$ ) and one old (mean $G=0.3$ ) network after learning four different stimuli. 
A

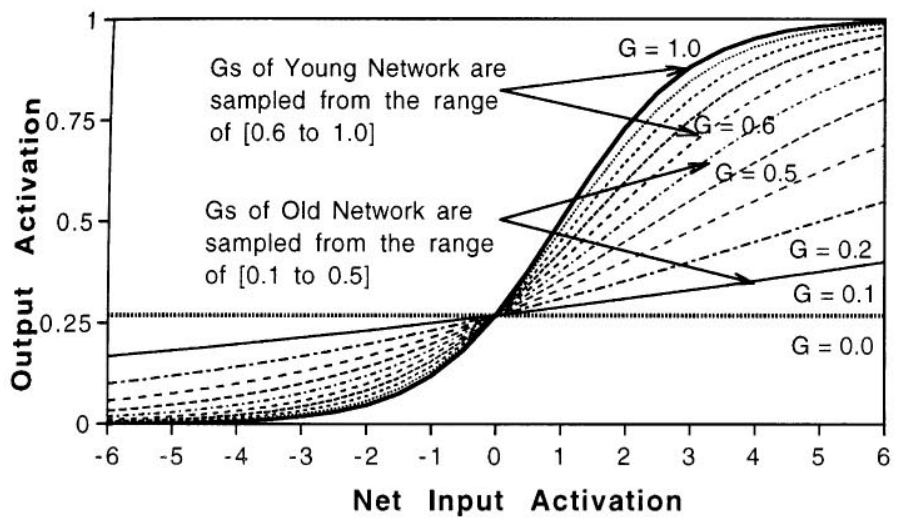

B

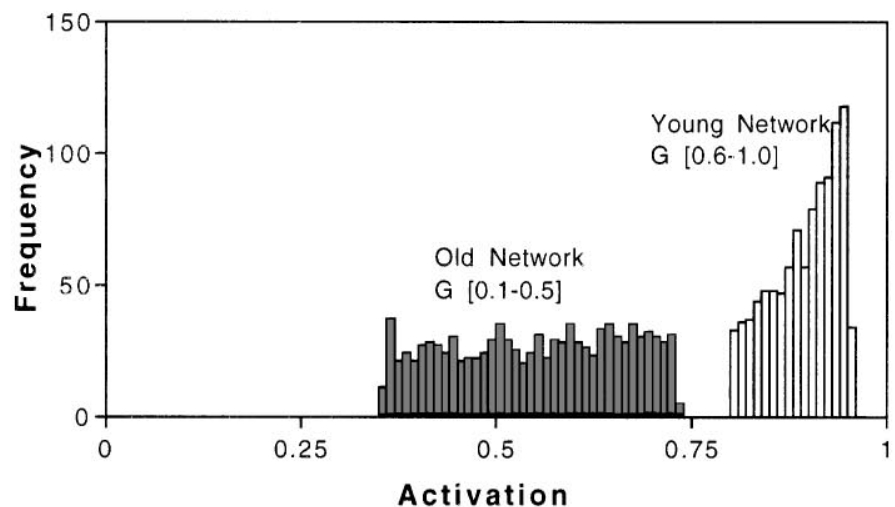

C
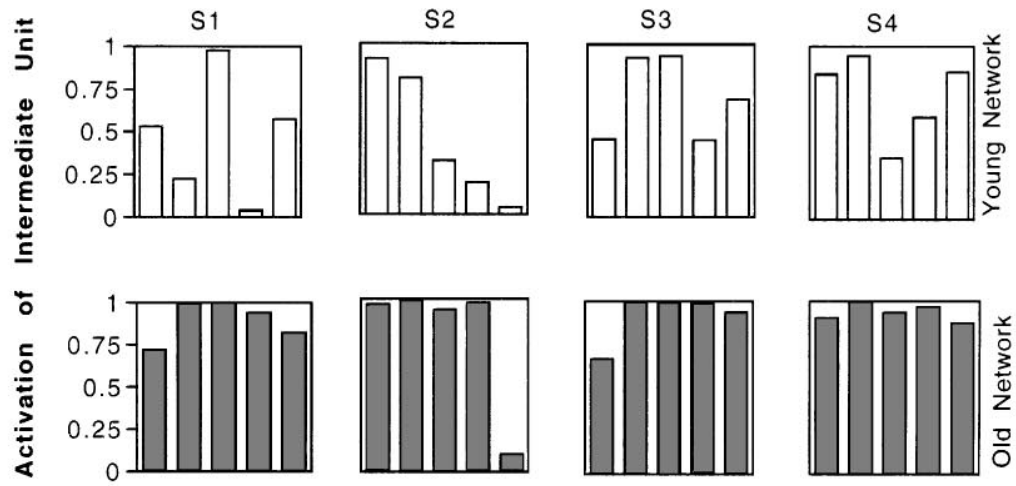

Units at the Intermediate Layer 


\section{Modeling age-related attenuation of catecholaminergic function}

Outside of cognitive aging research, there are quite a few formal models of neuromodulation [7]. However, to our knowledge no research effort has yet been devoted to instantiate formal models that link aging-induced changes in catecholaminergic modulation with a broad class of human cognitive aging phenomena at the behavioral level. In this study, we computationally investigate general principles of neural mechanisms linking catecholaminergic modulation of cortical neuron's responsivity to the distinctiveness of cortical representation and cognitive aging deficits.

Catecholamine's modulatory roles are manifold, depending on cortical regions, receptor types, and the techniques and concentrations of drug admission. In some cases, it has been found that catecholamine enhances a neuron's postsynaptic responsivity to afferent signal $[6,9]$. Or as suggested by more recent evidence, dopaminergic modulation in the PFC can be extremely precise, affecting a specific component of the PFC neuron's excitatory and inhibitory afferent input, without altering the neuron's general excitability [35]. These diversities notwithstanding, a general feature of catecholaminergic modulation can be more abstractly conceptualized as altering the signal-to-noise ratio of cortical information processing $[6,9,30]$. This general effect can be modeled by adjusting the gain parameter $(G)$ of the logistic activation function of processing units in feedforward backpropagation networks [30].

In previous studies, once the value of $G$ was reduced during the testing phase to simulate attenuated catecholaminergic function underlying cognitive deficits of schizophrenia [30] or increased to simulate the effect of D-amphetamine on mechanisms of selective attention [31], the same $G$ was applied to all units in the presumably affected module(s), and was kept constant across all processing steps. In order to incorporate evidence of random fluctuations in transmitter substance caused by probabilistic transmitter release $[14,15]$, we propose that rather than having a static $G$, each processing unit's $G$ is to be randomly sampled at each processing step during training and testing. Hence, the activation function of a unit is defined with a stochastic $G$ that varies its value slightly at each processing step:

$$
\left\{\begin{array}{l}
A_{i_{t}}=\frac{1}{1+\mathrm{e}^{-\left(G_{i t} \times \text { Net }_{i t}+\text { bias }\right)}} \\
\quad \text { with } G_{i_{t}} \in\left[G_{\min }, G_{\text {max }}\right], G_{\min }>0, \quad \operatorname{Net}_{i_{t}}=\sum_{j} W_{i j} I_{j}
\end{array}\right.
$$

where at each $t, I_{j}$ is the $j$ th input, and $W_{i j}$ is the connection weight.

Age-related decline in catecholaminergic function can then be simulated by reducing the mean of the uniform distribution from which the $G$ s of the units are sampled, while keeping the range of $G$ constant. 


\subsection{Reducing $G$ reduces responsivity, raises variability, and reduces representational distinctiveness}

Reducing mean $G$ flattens the logistic activation function, such that a unit's average responsivity is reduced (Fig. 1A). Subsequently, a $G$-reduced unit becomes less discriminative in responding to differences in inputs. In addition, reducing mean $G$ also increases the variability in the unit's output activation. Fig. 1B shows that for a given unit, reducing mean $G$ increases intra-network temporal variability. Similarly, when the $G$ s of different units in a network are randomly sampled, then the same magnitude of variability in $G$ s produces a greater amount of variability in output activation across units when the values of $G$ s are in a lower range. Conceptually, this implements a larger amount of random spatial variability of activation across units.

Note that a given amount of variability in $G$, simulating random fluctuations in transmitter substance, causes a greater amount of variability in output activation when the values of $G$ are in a lower range. It is a common view that cognitive aging deficits are associated with age-related increase in neuronal noise [34]; however, mechanisms for age-related increase in neuronal noise are not explicated. The interaction between the mean magnitude of $G$ and the effect of stochastic variation in $G$ on intra-network variability computationally depicts a potential neurochemical mechanism for agerelated increase in neural noise: as aging attenuates the efficacy of neuromodulation, random fluctuations in transmitter substances due to probabilistic transmitter release have a greater impact on the overall level of random variability in the aging brain.

Furthermore, reduced responsivity and increased intra-network variability subsequently affect the distinctiveness of the network's internal representations. To illustrate, activation patterns across the intermediate units of one "young" and one "old" network after learning different input-output pairs are plotted in Fig. 1C. Clearly, the activation patterns of the old network in response to different input signals are less distinctive than the activation patterns of the young network.

Together these results show that reduced responsivity of the units and increased intra-network variability as caused by mean $G$ reduction subsequently give rise to less differentiated (i.e., more inter-dependent) internal stimulus representations at the intermediate layer. One potential biological implication of this property is that as age-related catecholamine deficiency drives down cortical neurons' responsivity and raises the level of random variability during neural information processing, cortical representations elicited by different stimuli become less differentiable.

\section{Simulations linking catecholaminergic modulation with behavioral data}

The conjectured theoretical path from age-related decrement in catecholaminergic function to higher levels of random variability in the aging brain then to lessdistinctive cortical representation and cognitive aging deficits is examined with respect to multiple constraints from benchmark behavioral data. The effects of mean $G$ reduction in accounting for typical cognitive aging phenomena are tested in a series of simulations. To broadly sample different aspects of the behavioral data, the 
simulations include age effects on mean performance (see 4.1-4.3), variability in performance, and covariations between performances (see 4.4). All simulations involve two groups of otherwise identical networks that differed only in the mean values of their $G$ s (mean $G_{\text {young network }}=0.8$ and mean $G_{\text {old network }}=0.3$; for additional implementation details refer to $\mathrm{Li}$ and Lindenberger [19] and $\mathrm{Li}$ et al. [20].

\subsection{Aging, learning rate and asymptotic performance}

Memory research shows that the number of trials required for learning pairedassociates (i.e., arbitrary word pairs, such as computer-violin) increases dramatically with advancing age $[16,22]$. Fig. $2 \mathrm{~A}$ shows that, like the older subjects, the "old"

A

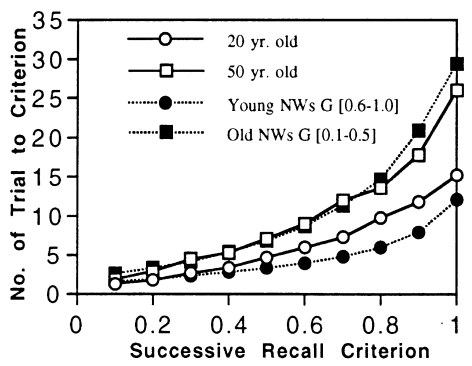

B

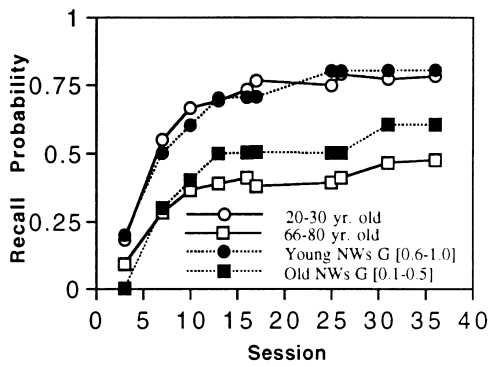

C

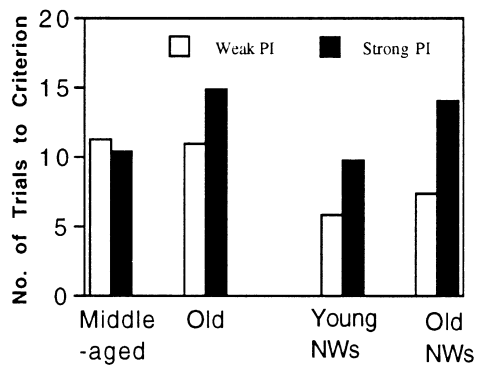

D

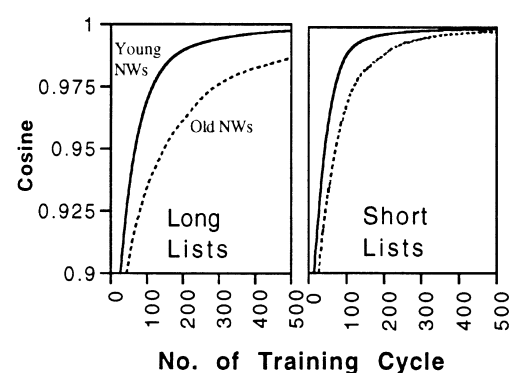

E

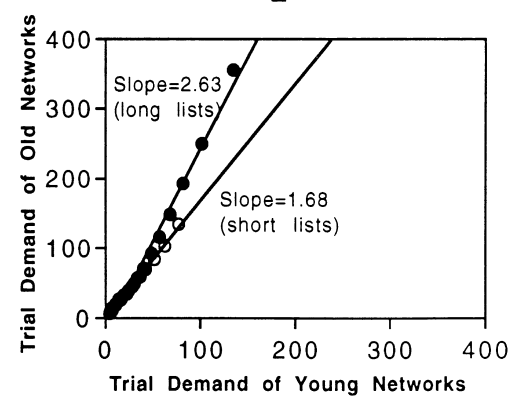


networks (i.e., with reduced mean $G$ ) require more learning trials than the young networks to reach increasingly strict recall criteria in paired-associate learning.

If old and young adults differ only in their learning rates, then one could expect that given enough practice old adults would eventually perform at the level of young adults. Alas, ample data of aging and practice effects on skill acquisition show that negative age differences often persist at asymptotes [4,27]. Fig. $2 \mathrm{~B}$ shows that negative age differences in learning paired-associates at asymptotes can also be accounted for by reducing mean $G$.

\subsection{Aging and interference susceptibility}

Another prominent cognitive aging deficit is older adults' increasing susceptibility to interference $[16,18]$. For instance, it is usually found that older adults are more susceptible to proactive interference (PI, the negative effect of previous learning on subsequent learning) than young people. Fig. $2 \mathrm{C}$ shows that while the number of trials required to learn the second list to criterion does not differ for middle-aged participants under conditions of weak and strong PI, old adults need many more trials to learn the second list when PI is strong. This age by degree of PI interaction can be simulated by the networks with high and low Gs. In line with the empirical data, the "old" networks show a disproportional increase in the number of trials required for learning the second list in the strong PI condition. This effect of mean $G$ reduction on increasing interference susceptibility has also been demonstrated in a multidimensional perceptual categorization paradigm [19].

\subsection{Aging and complexity cost}

One other robust empirical regularity of cognitive aging is the so-called age by complexity (or difficulty) effect, referring to the global pattern of an increase in the

Fig. 2. (A) Negative age effect on learning paired-associates in human subjects [22] and simulations. In neural network simulations it is often that one simulation cycle does not correspond exactly to one trial of human learning. For the results reported here, exchange factor is 17 (one human learning trial $=17$ network cycles). The successive recall criterion is from $10 \%$ to $100 \%$ correct for humans, and retrieved cosine from 0.90 to 0.99 for the networks. (B) Negative age difference in asymptotic performance in human subjects [4] and simulations. In this simulation, the exchange factor is 25 (one human session $=25$ network cycles, in the empirical study a session involves multiple learning trials). (C) Age-related increase in susceptibility to PI in dual-list paired-associate learning in human subjects [18] and simulations. Exchange factor used here was the same as Fig. 3A. The number of trials given for the first-list learning manipulated the extent of PI. For the weak and strong PI conditions, the networks were trained on the first list for 50 and 200 trials, respectively. The criterion of the second-list learning was an average of cosine $=0.95$ across different study lists. (D) Differences between the young and old learning curves as a function of task difficulty. Learning functions of young and old networks differed more in the more difficult condition (implemented here with longer list length; list length for the short and long lists were 3 and 8, respectively). (E) Young-old functions based on the learning curves presented in panel D. The slope of the young-old function is steeper in the condition with long lists, indicating a larger difference between the two groups of networks when learning the more difficult task. 

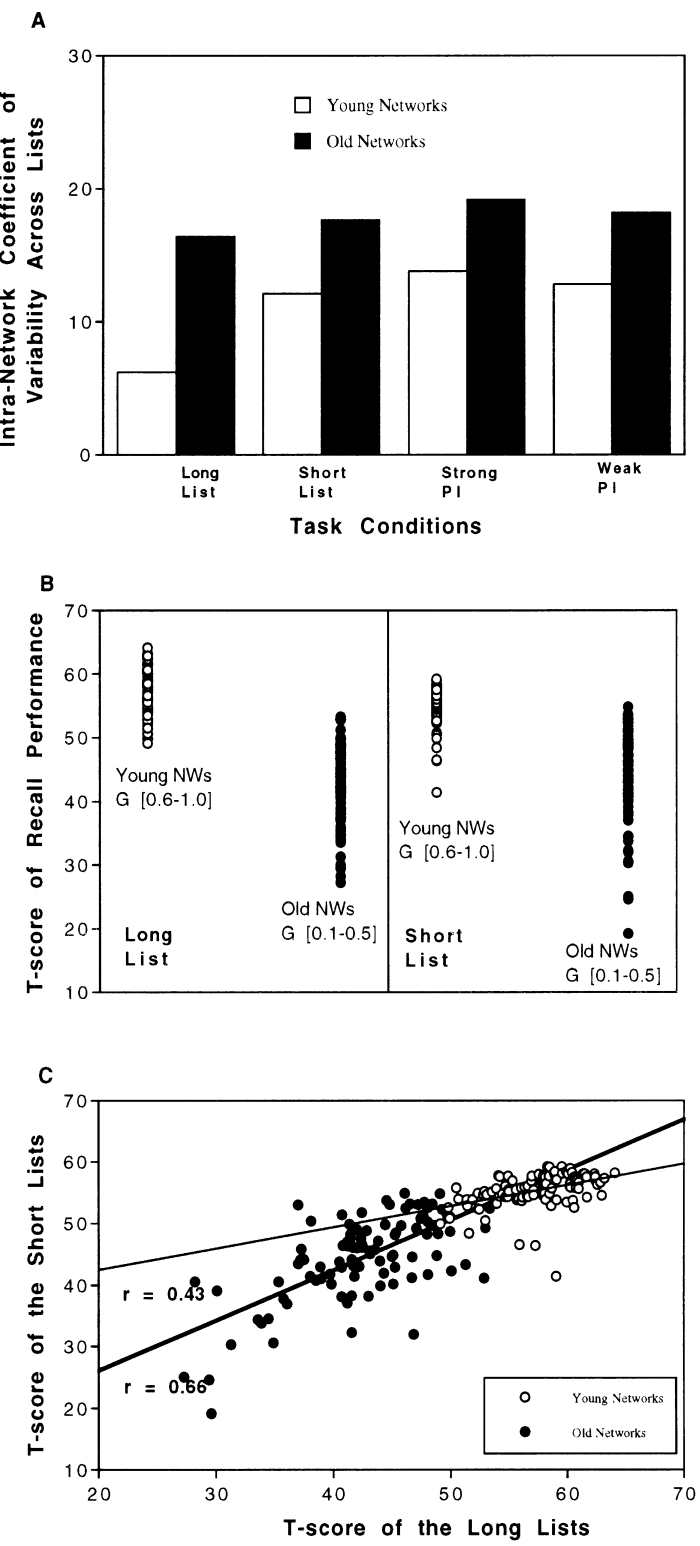

Fig. 3. (A) The effect of mean $G$ reduction on intra-network variability in performance level across different study lists in four conditions. (B) The effect of mean $G$ reduction on inter-network variability. Across conditions of list length, reducing mean $G$ not only reduces mean-level performance, but also increases inter-network variability. (In the condition with short lists, the SD of "young" and "old" networks are 2.85 and 5.62, respectively. In the condition with long lists, the SD of "young" and "old" networks are 3.51 and 6.92 , respectively). (C) The effect of mean $G$ reduction on covariation of performances. Reducing mean $G$ increases the correlation between performances with short and long lists. The correlation is stronger for the "old" than for the "young" networks ( $r=0.66$ vs. $r=0.43$, the difference between these two correlations is statistically significant, $z=2.4$ ). 
magnitude of negative age differences with increasing processing demands or task difficulty [17]. To test whether mean $G$ reduction can account for this broad class of phenomena, we simulated this effect within the paired-associate learning paradigm, using list length to manipulate task difficulty.

In line with the age by complexity effect, the difference between "young" and "old" networks is larger in the condition of long lists (Fig. 2D). A more detailed approach to examine the effect is to regress the number of trials required by the "old" networks to reach a range of successive performance criteria on the number of trials required by the "young" networks to reach the same criteria for both conditions. If indeed there is an age by complexity interaction, then the slope of the regression line for the more difficult condition should be steeper than that of the easier condition [17]. The corresponding young network-old network functions shown in Fig. 2E confirm the expectation.

\subsection{Aging, performance variability and covariation of performance}

In addition to negative age changes in levels, behavioral data also point to age-related increments in both intra-individual [11] and inter-individual [23] variability. To examine the effect of mean $G$ reduction on intra-network variability, the coefficient of variability was computed for each network based on its levels of performance across different study lists in the four conditions of paired-associates learning described earlier. Averages based on the coefficients of variability of each network were then computed for the "young" and "old" networks. Across all conditions, the average intra-network variability was larger in old networks (Fig. 3A).

With respect to examining the effect of mean $G$ reduction on inter-network variability, two groups of 100 networks were given the same amount of training to learn long and short lists of paired-associates. Performances of all networks were standardized across groups to a T-score metric (mean $=50, \mathrm{SD}=10$ ). Results presented in Fig. 3B show that across conditions of list length mean $G$ reduction not only lowers the mean levels of performance, but also raises inter-network variability.

Many psychometric studies conducted since the 1920s found that the correlation coefficients among subscales of intelligence tests are usually larger in older samples $[3,24]$. To examine the effect of mean $G$ reduction on the patterns of covariation, T-scores of 100 networks after learning the long lists were correlated for each of the two network groups with their own performances after learning the short lists. Fig. 3C shows that the correlation between the performances with long and short lists is stronger in "old" than in "young" networks (see [19] for additional related demonstrations with respect to other experimental paradigms).

\section{Conclusions}

We have demonstrated with a series of simulations that a single-parameter manipulation (i.e., reducing the mean $G$ of the activation function) accounts for a wide range of cognitive aging phenomena. Regarding unifying data and theories across levels, the 
simulations explicate a theoretical path from attenuated catecholaminergic function to reduced neural responsivity and increased neural noise to less distinct cortical representation in the aging brain to behavioral manifestations of age-related cognitive deficits. The interaction between the magnitude of $G$ and its random variability on intra-network variability illustrates a potential neurochemical mechanism for agerelated increase of neural noise involving the joint effect of age-related decline in neuromodulation and probabilistic transmitter release. Further theoretical efforts should aim at specifying formalisms that address the relationships between the distinctiveness of cortical representations and fundamental aspects of cognition, such as working memory capacity and processing speed. Regarding the integration of behavioral data across measurement dimensions, the demonstration that mean $G$ reduction simultaneously accounts for age-related differences in mean, variability, and covariation instantiates Spearman's [32] classical notion of general brain energy being the joint cause for individual differences in these three aspects of behavior, and the differentiation-dedifferentiation hypothesis of ability structure across the lifespan emerging from this notion $[3,24]$.

In this study, we focus on catecholaminergic modulation because of converging evidence with respect to its functional properties and age-related changes. However, the computational formalisms demonstrated here should generalize to other transmitter substances as long as they exhibit similar functional properties and age gradients.

\section{References}

[1] A.F.T. Arnsten, Catecholamine mechanisms in age-related cognitive decline, Neurobiol. Aging 14 (1993) 639-641.

[2] A.F.T. Arnsten, Catecholamine modulation of prefrontal cortical cognitive function, Trends Cog. Sci. 2 (1998) 436-447.

[3] P.B. Baltes, S.W. Cornelius, A. Spiro, J.R. Nesselroade, S.L. Willis, Integration versus differentiation of fluid/crystallized intelligence in old age, Dev. Psychol. 6 (1980) 625-635.

[4] P.B. Baltes, R. Kliegl, Further testing of the limits of cognitive plasticity: negative age differences in a mnemonic skill are robust, Dev. Psychol. 28 (1992) 121-125.

[5] Y. Burnod, H. Korn, Consequences of stochastic release of neurotransmitters of network computation in the central nervous system, Proc. Nat. Acad. Sci. USA 86 (1989) 352-356.

[6] L.A. Chiodo, T.W. Berger, Interactions between dopamine and amino-acid-induced excitation and inhibition in the striatum, Proc. Nat. Acad. Sci. USA 375 (1986) 198-203.

[7] J.M. Fellous, C. Linster, Computational models of neuromodulation, Neural Comput. 10 (1998) 771-805.

[8] C.E. Finch, Age-related changes in brain catecholamines: a synopsis of findings in C57BL/6J mice and other rodent models, Adv. Exp. Med. Biol. 113 (1978) 15-39.

[9] S.L. Foote, F.E. Freedman, A.P. Oliver, Effects of putative neurotransmitters on neuronal activity in monkey auditory cortex, Brain Res. 86 (1976) 229-242.

[10] W. Freeman, Non-linear gain mediating cortical stimulus-response relations, J. Biol. Cybernet. 33 (1979) 243-247.

[11] J.L. Fozard, J.C. Thomas, N.C. Waugh, Effects of age and frequency of stimulus repetitions on two-choice reaction time, J. Gerontol 38 (1976) 556-563.

[12] J.D.E. Gabrieli, Cognitive neuroscience of human memory, Annu. Rev. Psychol. 48 (1998) 87-115. 
[13] P.S. Goldman-Rakic, R.M. Brown, Regional changes of monoamines in cerebral cortex and subcortical structures of aging rehsus monkeys, Neuroscience 6 (1981) 177-187.

[14] N.A. Hessler, A.M. Shirke, R. Malinow, The probability of transmitter release at a mammalian central synapse, Nature 366 (1993) 569-572.

[15] B. Katz, The Release of Neural Transmitter Substances, Liverpool University Press, Liverpool, UK, 1965.

[16] D.H. Kausler, Learning and Memory in Normal Aging, Academic Press, New York, 1994.

[17] R. Kliegl, U. Mayr, R.T. Krampe, Time-accuracy functions for determining process and person differences: an application to cognitive aging, Cognitive Psychol. 26 (1994) 134-164.

[18] C.V. Lair, W.H. Moon, D.H. Kausler, Associative interference in the paired-associate learning of middle-aged and old subjects, Dev. Psychol. (1969) 548-552.

[19] S.-C. Li, U. Lindenberger, Cross-level unification: a computational exploration of the link between deterioration of neurotransmitter systems and dedifferentiation of cognitive abilities in old age, in: L.-G. Nilsson, M. Markowitsch (Eds.), Cognitive Neuroscience of Memory, Hogrefe \& Huber, Toronto, 1999, pp. 104-146.

[20] S.-C. Li, U. Lindenberger, P.A. Frensch, In search for an integrative framework of cognitive aging: a computational exploration of the link between processing efficiency and brain catecholamines, unpublished manuscript, 1996.

[21] P.G. MacRae, W.W. Spirduso, R.E. Wilcox, Reaction time and nigrostriatal dopamine function: the effect of age and practice, Brain Res. 451 (1988) 139-146.

[22] H.R. Monge, Studies of verbal learning from the college years through middle age, J. Gerontol. 26 (1971) 324-329.

[23] E.A. Nelson, D. Dannefer, Aged heterogeneity: fact or fiction? The fate of diversity in gerontological research, Gerontologist 32 (1992) 17-23.

[24] G. Reinert, Comparative factor analytic studies of intelligence throughout the human life span, in: L.R. Goulet, P.B. Baltes (Eds.), Life-span Developmental Psychology: Research and Theory, Academic Press, New York, 1970.

[25] T.A. Salthouse, Theoretical Perspectives on Cognitive Aging, LEA, Hillsdale, NJ, 1991.

[26] T.A. Salthouse, The processing-speed theory of adult age differences in cognition, Psychol. Rev. 103 (1996) 403-428.

[27] T.A. Salthouse, L.B. Somberg, Skilled performance: effects of adult age and experience on elementary processes, J. Exp. Psychol. 111 (1982) 176-207.

[28] T. Sawguchi, P.S. Goldman-Rakic, D1 dopamine receptors in prefrontal cortex: involvement in working memory, Science 251 (1991) 947-950.

[29] E.L. Schneider, J.W. Rowe, T.E. Johnson, N.J. Holbrook, H.J. Morrison (Eds.), Handbook of the Biology of Aging, Academic Press, New York, 1996.

[30] D. Servan-Schreiber, H. Printz, J.D. Cohen, A network model of catecholamine effects: gain, signalto-noise ratio, and behavior, Science 249 (1990) 892-895.

[31] D. Servan-Schreiber, R.M. Bruno, C.S. Carter, J.D. Cohen, Dopamine and the mechanisms of cognition: Part I. A neural network model predicting dopamine effects on selective attention, Biol. Psychiatry 43 (1998) 713-722.

[32] C.E. Spearman, The Abilities of Man, MacMillan, New York, 1927.

[33] N.D. Volkow et al., Association between decline in brain dopamine activity with age and cognitive and motor impairment in healthy individuals, American J. of Psychiatry 155 (1998) 344-349.

[34] A.T. Welford, Performance, biological mechanisms and age: a theoretical sketch, in: A.T. Welford, J.E. Birren (Eds.), Behavior, Aging, and the Nervous System, Thomas, Springfield, IL, 1965, pp. 3-20.

[35] G.V. Williams, P.S. Goldman-Rakic, Blockage of dopamine D1 receptors enhances memory fields of prefrontal neurons in primate cerebral cortex, Nature 376 (1995) 572-575. 


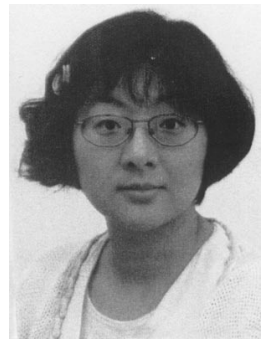

Shu-Chen $\mathbf{L i}$ is a research scientist at the Max Planck Institute for Human Development, Berlin, Germany. She received her Ph.D. from the University of Oklahoma, USA, in December 1994, and was a postdoctoral fellow at McGill University, Montreal, Canada. Her research stretches across behavioral studies of lifespan cognitive development, cognitive neuroscience and computational modeling. She is interested in modeling behavioral data of cognitive performance by computationally implementing neurobiological mechanisms that may have bearings on information-processing mechanism, such as processing speed, inhibition and working memory. She had also published empirical papers on serialorder memory and theoretical articles on using parameter sensitivity analyses as a diagnostic tool for model comparison. She thanks especially Prof. Paul B. Baltes and the Max Planck Institute for sponsoring this project.

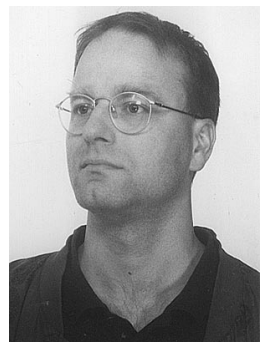

Ulman Lindenberger is a research scientist at the Max Planck Institute for Human Development, Berlin. He co-directs several research projects on lifespan cognitive development, and serves on the editorial board of several international journals in the field of cognitive aging. He received his Ph.D. and his habilitation at the Free University of Berlin in 1990 and 1998, respectively. His general research interests are in the field of lifespan cognitive development. His specific interests include the causes and dimensionality of negative age changes in adult cognition, the relationship between age changes in sensory and cognitive functioning, as well as issues regarding experimental and multivariate methodology.

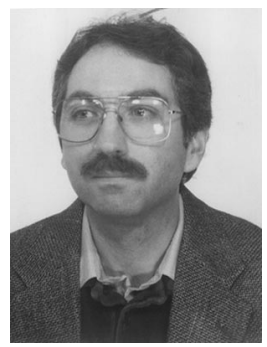

Peter A. Frensch studied electrical engineering, psychology, and philosophy at the Universities of Darmstadt and Trier, Germany, and at Yale University, where he received his M.S., M.Phil., and Ph.D. He worked as an assistant and associate professor in the department of Psychology at the University of Missouri-Columbia and as a senior research scientist at the Max-Plank-Institute for Human Development in Berlin, Germany. Since 1998, he has been a full professor in the department of Psychology at Humboldt-University, Berlin, Germany. He is coordinating editor of the journal Psychological Research. His research interests include human learning, memory, and problem solving. 